\title{
SOCIALLY OPTIMAL COORDINATION: CHARACTERIZATION AND POLICY IMPLICATIONS
}

George-Marios Angeletos

Alessandro Pavan

Working Paper 12778

http://www.nber.org/papers/w12778

\author{
NATIONAL BUREAU OF ECONOMIC RESEARCH \\ 1050 Massachusetts Avenue \\ Cambridge, MA 02138
}

December 2006

This paper was prepared for the 2006 meeting of the European Economic Association in Vienna. We are grateful to NSF for financial support (collaborative research grants SES-0519069 and SES-0518810). The views expressed herein are those of the author(s) and do not necessarily reflect the views of the National Bureau of Economic Research.

(C) 2006 by George-Marios Angeletos and Alessandro Pavan. All rights reserved. Short sections of text, not to exceed two paragraphs, may be quoted without explicit permission provided that full credit, including $\odot$ notice, is given to the source. 
Socially Optimal Coordination: Characterization and Policy Implications

George-Marios Angeletos and Alessandro Pavan

NBER Working Paper No. 12778

December 2006

JEL No. C72,D62,D82,E3,E5

\begin{abstract}
$\underline{\text { ABSTRACT }}$
In recent years there has been a growing interest in macro models with heterogeneity in information and complementarity in actions. These models deliver promising positive properties, such as heightened inertia and volatility. But they also raise important normative questions, such as whether the heightened inertia and volatility are socially undesirable, whether there is room for policies that correct the way agents use information in equilibrium, and what are the welfare effects of the information disseminated by the media or policy makers. We argue that a key to answering all these questions is the relation between the equilibrium and the socially optimal degrees of coordination. The former summarizes the private value from aligning individual decisions, whereas the latter summarizes the value that society assigns to such an alignment once all externalities are internalized.
\end{abstract}

George-Marios Angeletos

Department of Economics

MIT

50 Memorial Drive, E52-251

Cambridge, MA 02142

and NBER

angelet@mit.edu

Alessandro Pavan

Northwestern University

Department of Economics

2001 Sheridan Road

Arthur Andersen Hall 3239

Evanston, IL 60208

alepavan@northwestern.edu 


\section{Introduction}

In recent years, there has been a growing interest in models that share the following two key features: (i) heterogeneity in information about the underlying economic fundamentals; and (ii) complementarity in actions. Examples include the beauty-contest game in Morris and Shin (2002); the investment games in Angeletos and Pavan (2004) and Angeletos, Lorenzoni and Pavan (2006); the common-interest game in the paper by Morris and Shin in this issue; and the business-cycle models in Woodford (2002), Hellwig (2005), Lorenzoni (2005), and Roca (2005). ${ }^{1}$

These models deliver interesting positive properties, such as inertia (i.e., slow response to changes in the underlying fundamentals) and heightened non-fundamental volatility (i.e., high sensitivity to common noise in information about the underlying fundamentals). But they also raise important normative questions.

1. Is the heightened inertia or volatility due to complementarity socially undesirable?

2. Are there policies that could manipulate the way agents use information, and thereby correct the sensitivity of the equilibrium to both fundamentals and noise? If yes, how do these policies look like?

3. How does the incompleteness of information affect welfare? What is the social value of the information disseminated by prices, market experts, or the media? Should central banks disclose the information they collect and the forecasts they make about the economy in a transparent and timely fashion, or is there room for "constructive ambiguity"?

To answer these questions, one needs: (1) to compare the equilibrium use of information with an appropriate constrained efficiency benchmark, namely the use of available information that maximizes welfare; $(2)$ to identify policies that implement the efficient use of information as an equilibrium; and (3) to understand the comparative statics of equilibrium welfare with respect to the information structure.

Ongoing work (Angeletos and Pavan, 2006a,b) undertakes these tasks in a broad class of economies with heterogeneous information, externalities, and strategic complementarity or substitutability in actions, and discusses a variety of applications. In this

\footnotetext{
${ }^{1}$ This class of models differs from global games in that the coordination element is moderate enough that the equilibrium is unique no matter the precision of private and public information. Related are also models with "inattentive" agents, as in Mankiw and Reis (2006).
} 
paper, we restrict attention to a (sub)class in which inefficiency emerges only when information is incomplete, thus isolating the inefficiencies that originate in the use of information from other possible distortions. ${ }^{2}$

This facilitates the main message of this paper: the key to answering all the questions above is the relation between the equilibrium and the socially optimal degrees of coordination. The former is identified with the slope of an agent's best response with respect to others' activity, and pins down the equilibrium use of information; the latter is identified with the slope that would make agents internalize all externalities, and pins down the efficient use of information. The former summarizes the private value from aligning individual decisions; the latter summarizes the value that society assigns to such an alignment.

\section{A simple model}

There is a continuum of agents, indexed by $i$ and uniformly distributed over $[0,1]$, each choosing an action $k_{i} \in \mathbb{R}$ (e.g., think of $k$ as investment). There is also a government, which makes transfers $t_{i}$ to the agents, subject to budget balance, $\int t_{i} d i=0$.

Payoffs. Agent $i$ 's payoff is $u_{i}+t_{i}$, where

$$
u_{i}=-\left(k_{i}-\theta\right)^{2}-r\left(L_{i}-\bar{L}\right)-r^{*} \bar{L} .
$$

$\theta \in \mathbb{R}$ is an exogenous random fundamental (e.g., aggregate productivity), $L_{i}=$ $\int\left(k_{j}-k_{i}\right)^{2} d j$ is the mean-square distance of $i$ 's action from other agents' actions, $\bar{L}=\int L_{i} d i$ is the average of these distances, and $r$ and $r^{*}$ are non-negative scalars.

This payoff structure has a simple interpretation. The term $\left(k_{i}-\theta\right)^{2}$ captures the value of taking an action that is aligned with the fundamentals, whereas the term $L_{i}$ introduces a private value to aligning one's action to those of others - the source of strategic complementarity. The term $\bar{L}$, on the other hand, introduces an externality which controls the discrepancy, if any, between the private and the social value of such alignment.

\footnotetext{
${ }^{2}$ Actually, the results presented here extend to economies where the distance between the completeinformation equilibrium and the first-best is non-zero, as long as this distance is invariant with $\theta$ (see Angeletos and Pavan, 2006a,b.) Thus, one can think of the class considered here as capturing businesscycle models in which, under complete information, the "output gap" is non-zero but constant over the business cycle.
} 
Indeed, since $\bar{L}$ does not depend on agent $i$ 's action, from a strategic viewpoint it is as if payoffs were $u_{i}^{\text {private }}=-\left(k_{i}-\theta\right)^{2}-r L_{i}$. Aggregate welfare, on the other hand, is given by

$$
w \equiv \int u_{i} d i=\int\left[-\left(k_{i}-\theta\right)^{2}-r^{*} L_{i}\right] d i
$$

Hence, from a social perspective it is as if payoffs were given by $u_{i}^{\text {social }}=-\left(k_{i}-\right.$ $\theta)^{2}-r^{*} L_{i}$. In this sense, $r$ parametrizes the private value of aligning choices, while $r^{*}$ parametrizes the social value of such alignment.

Remark. While the particular payoffs assumed here admit a convenient interpretation, the key assumption is only that inefficiency vanishes once information is complete (Angeletos and Pavan, 2006a,b). Here, the complete-information equilibrium and the first-best allocation are both given by $k_{i}=\theta, \forall i{ }^{3}$

Information. For the purposes of this paper it is important to allow agents to have heterogeneous information about $\theta$; for concreteness, we adopt a Gaussian specification. Before agents move, nature draws $\theta$ from a Normal distribution with mean $\mu$ and variance $\sigma_{\theta}^{2}$. The realization of $\theta$ is not observed by the agents. Instead, agents observe private signals $x_{i}=\theta+\xi_{i}$ and a public signal $y=\theta+\varepsilon$, where $\xi^{i}$ and $\varepsilon$ are, respectively, idiosyncratic and common Normal noises, independent of one another as well as of $\theta$, with variances $\sigma_{x}^{2}$ and $\sigma_{y}^{2}$.

\section{Equilibrium degree of coordination}

We start by characterizing equilibrium without government intervention $\left(t_{i}=0\right.$ for all i); we reintroduce the government in Section 5 .

The best response of agent $i$ solves $\partial \mathbb{E}_{i} u_{i} / \partial k_{i}=0$, which reduces to

$$
k_{i}=(1-\alpha) \mathbb{E}_{i} \theta+\alpha \mathbb{E}_{i} K
$$

where $\alpha=r /(1+r)$ and $K=\int k_{j} d j$. That is, the best response of an agent is a weighted average of his expectation of $\theta$ and his expectation of aggregate activity $K{ }^{4}$ The equilibrium is then given by the fixed point to this best-response condition.

\footnotetext{
${ }^{3}$ Also note that the payoff structure assumed here is nested in the more general one considered in Angeletos and Pavan $(2006 \mathrm{a}, \mathrm{b})$ by rewriting payoffs as $u_{i}=U\left(k_{i}, K, \sigma_{k}^{2}, K\right)=-(1+r) k_{i}^{2}+2 k_{i} \theta+$ $2 r k_{i} K-\left(2 r^{*}-r\right) \sigma_{k}^{2}-r K^{2}-\theta^{2}$, where $K \equiv \int k_{j} d j$ is the mean, and $\sigma_{k}^{2} \equiv \int\left(k_{j}-K\right)^{2} d j$ the dispersion, of activity in the population.

${ }^{4}$ For any random variable $z$, we let $\mathbb{E}_{i} z \equiv \mathbb{E}_{i}\left[z \mid x_{i}, y\right]$.
} 
The slope of best responses with respect to aggregate activity $K$, which here is a simple increasing function of $r$, captures how much agents care to align their actions with one another; it summarizes how the private value to coordination impacts equilibrium behavior. We accordingly call $\alpha$ the equilibrium degree of coordination.

This coefficient plays a key role on how agents use information in equilibrium. When $\alpha=0$, condition (1) reduces to $k_{i}=\mathbb{E}_{i} \theta=\lambda_{\mu} \mu+\lambda_{y} y+\lambda_{x} x_{i}$, where $\left(\lambda_{\mu}, \lambda_{y}, \lambda_{x}\right)$ are the familiar Bayesian weights. ${ }^{5}$ That is, when $\alpha=0$, the equilibrium action is simply the best predictor of $\theta$. When instead $\alpha>0$, the equilibrium is given by the linear strategy

$$
k_{i}=\gamma_{\mu} \mu+\gamma_{y} y+\gamma_{x} x_{i}
$$

where the coefficients $\left(\gamma_{\mu}, \gamma_{y}, \gamma_{x}\right)$ are given by

$$
\gamma_{\mu}=\frac{\lambda_{\mu}}{1-\alpha \lambda_{x}}>\lambda_{\mu}, \quad \gamma_{y}=\frac{\lambda_{y}}{1-\alpha \lambda_{x}}>\lambda_{y}, \quad \gamma_{x}=\frac{(1-\alpha) \lambda_{x}}{1-\alpha \lambda_{x}}<\lambda_{x}
$$

That is, a positive degree of coordination increases the reliance of equilibrium actions to the prior and to public information, and decreases the reliance to private information. ${ }^{6}$

The logic for this result is simple. The prior and the public signal are relatively better predictors of others' activity than the private signal. The higher $\alpha$, the more agents care to align their choices, and hence the more they find it optimal to rely on $\mu$ and $y$. It follows that both $\gamma_{\mu}$ and $\gamma_{y}$ increase with $\alpha$, whereas $\gamma_{x}$ decreases with $\alpha$.

The equilibrium use of information in turn determines how aggregate activity responds to both fundamentals and noise. Using $\gamma_{\mu}+\gamma_{x}+\gamma_{y}=1$ and $y=\theta+\varepsilon$, we can write aggregate activity as $K=\gamma_{\mu} \mu+\left(1-\gamma_{\mu}\right) \theta+\gamma_{y} \varepsilon$, where $\varepsilon$ is common noise. It follows that a higher $\alpha$, by increasing $\gamma_{\mu}$ and $\gamma_{y}$, reduces the sensitivity of aggregate activity to the fundamental and increases its sensitivity to common noise. That is, a higher degree of coordination increases both inertia and volatility. At the same time, because a higher $\alpha$ reduces the reliance on private information, and hence the sensitivity to idiosyncratic noise, it also reduces the dispersion of activity in the cross-section of the population.

\footnotetext{
${ }^{5}$ That is, $\lambda_{\mu} \equiv \sigma_{\theta}^{-2} / \sigma^{-2}, \lambda_{y} \equiv \sigma_{y}^{-2} / \sigma^{-2}$, and $\lambda_{x} \equiv \sigma_{x}^{-2} / \sigma^{-2}$, where $\sigma^{-2} \equiv \sigma_{\theta}^{-2}+\sigma_{y}^{-2}+\sigma_{x}^{-2}$.

${ }^{6}$ See the Appendix for the derivation of (2) and (3).
} 


\section{Socially optimal degree of coordination}

We next turn to the characterization of a particular constrained efficient allocation. This allocation is the strategy that maximizes ex-ante welfare (i.e., expected utility behind the veil of ignorance) taking as given the dispersion of information in the population. It can be represented as the solution to a planner's problem, where the planner can perfectly control how each agent uses his available information, but can not transfer information from one agent to another.

As it turns out, the efficient allocation is the strategy that satisfies

$$
k_{i}=\left(1-\alpha^{*}\right) \mathbb{E}_{i} \theta+\alpha^{*} \mathbb{E}_{i} K,
$$

where $\alpha^{*}=2 r^{*} /\left(1+2 r^{*}\right)$. Condition (4) is the analog of (1) with $\alpha^{*}$ replacing $\alpha$. This suggests a simple interpretation of condition (4). The efficient allocation can be implemented by manipulating the equilibrium degree of coordination perceived by the agents (for example, through taxes, as we will see in the next section). The coefficient $\alpha^{*}$ then summarizes how much the planner wants the agents to align their actions. We accordingly identify $\alpha^{*}$ with the (socially) optimal degree of coordination. ${ }^{7}$

Just as $\alpha$ pins down the equilibrium use of information, $\alpha^{*}$ pins down the efficient use of information: the efficient allocation is given by

$$
k_{i}=\gamma_{\mu}^{*} \mu+\gamma_{y}^{*} y+\gamma_{x}^{*} x_{i},
$$

where the coefficients $\left(\gamma_{\mu}^{*}, \gamma_{y}^{*}, \gamma_{x}^{*}\right)$ are as in (3) replacing $\alpha$ with $\alpha^{*}$. By implication, the discrepancy, if any, between the equilibrium and the efficient use of information is determined merely by the discrepancy, if any, between $\alpha$ and $\alpha^{*}$ : the sensitivity of the equilibrium allocation to the prior mean and to public information is inefficiently high if and only if $\alpha>\alpha^{*}$. The answer to the first question raised in the introduction thus reduces to a simple comparison between $\alpha$ and $\alpha^{*}$.

Result 1. If $\alpha>\alpha^{*}$, then the inertia and the volatility featured in equilibrium are inefficiently high; welfare would be higher if agents were to perceive a lower complementarity in their actions. But if $\alpha \leq \alpha^{*}$, then the heightened levels of inertia and volatility featured in equilibrium are anything but excessive.

\footnotetext{
${ }^{7}$ See the Appendix for the derivation of condition (4).A similar condition characterizes the efficient use of information in the richer class of economies considered in Angeletos and Pavan (2006a). In general, the mapping from the underlying payoff structure to the coefficient $\alpha^{*}$ is not a simple as here, but it remains true that $\alpha^{*}$ summarizes the social value of aligning choices across agents.
} 


\section{Optimal policy}

When the equilibrium use of information is inefficient $\left(\alpha \neq \alpha^{*}\right)$, a novel role for policy emerges: welfare can be enhanced with policies that manipulate the agents' incentives to align their decisions. In our framework, this can be achieved with a relatively simple linear tax system, which is the incomplete-information analogue of a Pigou tax system - the key is to make the tax rate contingent on ex-post aggregate activity.

Consider the following tax scheme. Transfers take place at the end of the game, once agents have made their choices; at that point, the government either observes $\theta$ directly, or it infers it by observing $K$ and $y$. The transfer made to agent $i$ is then given by

$$
t_{i}=-\tau(K, \theta) k_{i}+T(K, \theta)
$$

where $\tau(K, \theta)$ is the marginal tax rate and $T(K, \theta)$ a lump-sum transfer. The tax rate is given by

$$
\tau(K, \theta)=(2+2 r)\left(\tau_{K} K+\tau_{\theta} \theta\right)
$$

for some $\tau_{K}, \tau_{\theta} \in \mathbb{R}$; the coefficients $\tau_{K}$ and $\tau_{\theta}$ parametrize the sensitivity of the tax rate to aggregate activity and to the fundamental, while the term $2+2 r$ is just a normalization. Finally, budget balance imposes $T(K, \theta)=\tau(K, \theta) K$.

Agent $i$ anticipates that the tax rate he will pay per unit of $k_{i}$ will depend on aggregate activity $K$. His best response is thus given by

$$
k_{i}=\left(1-\alpha-\tau_{\theta}\right) \mathbb{E}_{i} \theta+\left(\alpha-\tau_{K}\right) \mathbb{E}_{i} K
$$

It follows that the proposed policy implements the efficient allocation as an equilibrium if and only if $\tau_{K}=\alpha-\alpha^{*}=-\tau_{\theta}$. Hence, the optimal contingency of $\tau$ on $K$ is dictated by the difference between $\alpha$ and $\alpha^{*}$.

Result 2. Any inefficiency in the inertia or volatility of the equilibrium allocation can be corrected by appropriately designing the contingency of the marginal tax rate on aggregate activity. The optimal tax rate must increase with $K$ if and only if $\alpha>\alpha^{*}$. 


\section{Social value of information}

We now show how the relation between $\alpha$ and $\alpha^{*}$ helps understand the comparative statics of equilibrium welfare with respect to information. ${ }^{8}$

We find it useful to decompose any change in the information structure into an accuracy and a commonality effect. We identify the accuracy of available information with the reciprocal of the total noise in the agents' forecasts of the fundamental, and its commonality with the correlation of noise across agents. That is, letting $\omega_{i} \equiv$ $\theta-\mathbb{E}_{i} \theta$ denote agent $i$ 's forecast error, we define accuracy as $\sigma^{-2}=1 / \operatorname{Var}\left(\omega_{i}\right)$ and commonality as $\delta=\operatorname{Corr}\left(\omega_{i}, \omega_{j}\right)$, for $i \neq j .^{9}$

Welfare is lower under incomplete than under complete information because the noise in the agents' information induces "errors" in their actions relative to what they would have done if they knew $\theta$. These errors manifest themselves in two dimensions. First, common noise (i.e., noise in public information) generates non-fundamental volatility, that is, variation in aggregate activity $K$ relative to the complete-information level $\theta$. Second, idiosyncratic noise (i.e., noise in private information) generates crosssectional dispersion, that is, variation in individual activity $k$ across agents. Both types of errors contribute to lower welfare.

An increase in accuracy for given commonality - a reduction in total noise for given composition of noise - reduces both types of errors and hence necessarily increases welfare. An increase in commonality for given accuracy, on the other hand, substitutes one type of error for another: in equilibrium it decreases dispersion but can increase volatility. Whether this increases welfare depends again on the relation between the equilibrium and the socially optimal degree of coordination.

Result 3. (i) Equilibrium welfare necessarily increases with the accuracy of information. (ii) If $\alpha \leq \alpha^{*}$, welfare also increases with commonality; if instead $\alpha>\alpha^{*}$, welfare is non-monotonic in commonality.

To understand part (ii), note that, when the planner chooses the optimal degree of coordination, he effectively faces a trade off between dispersion and volatility: the higher the degree of coordination perceived by the agents, the lower the sensitivity

\footnotetext{
${ }^{8}$ We focus on equilibrium without government. Since the optimal policy restores any efficiency in the equilibrium use of information, the welfare effects of information in an economy with optimal policy coincide with those in an economy where $\alpha=\alpha^{*}$.

${ }^{9}$ It is easy to check that $\sigma^{-2}=\sigma_{\theta}^{-2}+\sigma_{y}^{-2}+\sigma_{x}^{-2}$, while $\delta=\left(\sigma_{\theta}^{-2}+\sigma_{y}^{-2}\right) / \sigma^{-2}$.
} 
to idiosyncratic noise and the higher the sensitivity to common noise, and hence the lower the dispersion and the higher the volatility. It follows that the optimal degree of coordination reflects social preferences over dispersion and volatility: a higher $\alpha^{*}$ means a higher willingness to substitute dispersion for volatility.

When the equilibrium use of information is efficient $\left(\alpha=\alpha^{*}\right)$, higher commonality, by substituting dispersion for volatility, necessarily raises welfare (provided $\alpha^{*}>0$, so that there is a strictly positive value to aligning choices). When, instead, the equilibrium use of information is inefficient $\left(\alpha \neq \alpha^{*}\right)$, the welfare effect of commonality depends on its effect on this inefficiency. Intuitively, an increase in commonality always facilitates a closer alignment of decisions, but whether this improves efficiency depends on whether there is too little or too much alignment to start with. When $\alpha<\alpha^{*}$, higher commonality mitigates the inefficiency and therefore necessarily raises welfare. When instead $\alpha>\alpha^{*}$, higher commonality exacerbates the inefficiency and may thereby reduce welfare.

Having understood the social value of accuracy and commonality, it is now easy to understand the welfare effects of any change in information. For example, suppose that a prompt release of news by the media, more transparency in central-bank communications, or more timely publication of macroeconomic statistics by the administration, result in an increase in the precision of available public information, keeping constant the precision of private information. This induces an increase in both the accuracy and the commonality of information. By Result 3, the increase in accuracy necessarily boosts welfare; but the increase in commonality can decrease welfare if the equilibrium degree of coordination is inefficiently high. The following is then an immediate implication.

Result 3b. More precise public information necessarily increases welfare if $\alpha \leq \alpha^{*}$, but can decrease welfare if $\alpha$ is sufficiently higher than $\alpha^{*}$.

As another example of how the relation between $\alpha$ and $\alpha^{*}$ affects the social value of information, suppose that a policy maker faces the choice between two possible ways of communicating to the market: very fine messages that convey a lot of information but-precisely because they are too fine - are likely to be interpreted in idiosyncratic ways; and simpler messages that convey less information but-precisely because they are simpler - admit a common interpretation. Then, the policy maker effectively faces a trade-off between accuracy and commonality; if $\alpha^{*} \geq \alpha$, so that commonality is socially 
desirable, he may well opt for the coarser messages. (See Morris and Shin (2006) for a motivation and further implications.)

\section{Conclusion}

We argued that the relation between the private and the social value to coordination is the key to answering all the normative questions raised in the introduction-whether the inertia and volatility featured in equilibrium are inefficient, what is the role of policy in correcting how agents use information, and what is the social value of information.

We illustrated this point within the context of a specific example, which admitted a simple parametrization of the private and social values of coordination. In general, the mapping from the payoff structure to the equilibrium and optimal degrees of coordination need not be as simple as in the example considered here. Yet, the main insight extends: the private value of aligning choices can be read from the slope of best responses, while the social value of such alignment can be read from the slope of best responses that would make agents internalize all externalities. (See Angeletos and Pavan, 2006a.)

For example, in the beauty-contest models of Morris and Shin (2002) and Angeletos, Lorenzoni and Pavan (2006), the complementarity perceived by the agents is not warranted from a social perspective $\left(\alpha>0\right.$ but $\left.\alpha^{*}=0\right)$. This explains why in these models welfare may decrease with higher commonality, and hence may also decrease with more precise public information. In contrast, in the business-cycle models of Hellwig (2005) and Roca (2005), the social value of coordination turns out to be higher than the private one $\left(\alpha^{*}>\alpha\right)$. This is because individual utility falls with cross-sectional dispersion in prices - an externality that raises the social value of aligning prices across firms. As a result, the heightened inertia and volatility featured in these models due to the complementarity in pricing decisions are anything but excessive; and welfare necessarily increases with more precise public information. 


\section{Appendix}

Proof of Conditions (2)-(3). Suppose that all agents follow a linear strategy as in (2), for some coefficients $\left(\gamma_{\mu}, \gamma_{y}, \gamma_{x}\right)$. Given this strategy, aggregate activity is given by $K=\gamma_{\mu} \mu+\gamma_{y} y+\gamma_{x} \theta$ and the best-response condition (1) for agent $i$ reduces to

$$
\begin{aligned}
k_{i} & =(1-\alpha) \mathbb{E}_{i}[\theta]+\alpha \mathbb{E}_{i}\left[\gamma_{\mu} \mu+\gamma_{y} y+\gamma_{x} \theta\right] \\
& =\left(1-\alpha+\alpha \gamma_{x}\right) \mathbb{E}_{i}[\theta]+\alpha \gamma_{\mu} \mu+\alpha \gamma_{\mu} y \\
& =\left[\left(1-\alpha+\alpha \gamma_{x}\right) \lambda_{\mu}+\alpha \gamma_{\mu}\right] \mu+\left[\left(1-\alpha+\alpha \gamma_{x}\right) \lambda_{y}+\alpha \gamma_{y}\right] y+\left[\left(1-\alpha+\alpha \gamma_{x}\right) \lambda_{x}\right] x_{i} .
\end{aligned}
$$

For the proposed strategy to be an equilibrium, it must be that

$$
\begin{aligned}
\gamma_{\mu} & =\left(1-\alpha+\alpha \gamma_{x}\right) \lambda_{\mu}+\alpha \gamma_{\mu} \\
\gamma_{y} & =\left(1-\alpha+\alpha \gamma_{x}\right) \lambda_{y}+\alpha \gamma_{y} \\
\gamma_{x} & =\left(1-\alpha+\alpha \gamma_{x}\right) \lambda_{x} .
\end{aligned}
$$

Solving the above for $\left(\gamma_{\mu}, \gamma_{y}, \gamma_{x}\right)$ gives (3). (For a discussion of when this linear strategy is the unique equilibrium, see Angeletos and Pavan, 2006a.)

Proof of Condition (4). Consider an arbitrary strategy $k: \mathbb{R}^{2} \rightarrow \mathbb{R}$. Ex-ante welfare is given by

$$
\mathbb{E} w=\int_{(\theta, y)}\left\{\int_{x_{i}}\left[-\left(k_{i}-\theta\right)^{2}-r^{*} L_{i}\right] d F\left(x_{i} \mid \theta, y\right)\right\} d G(\theta, y)
$$

where $F(x \mid \theta, y)$ is the c.d.f. of the conditional distribution of $x$ given $(\theta, y), G(\theta, y)$ is the c.d.f. of the joint distribution of $(\theta, y), L_{i}=\int_{x_{j}}\left(k_{i}-k_{j}\right)^{2} d F\left(x_{j} \mid \theta, y\right), k_{i}=$ $k\left(x_{i}, y\right)$, and $k_{j}=k\left(x_{j}, y\right)$. Note that

$$
\begin{aligned}
\int_{x_{i}} L_{i} d F\left(x_{i} \mid \theta, y\right) & =\int_{x_{i}} \int_{x_{j}}\left(k_{i}^{2}+k_{j}^{2}-2 k_{i} k_{j}\right) d F\left(x_{j} \mid \theta, y\right) d F\left(x_{i} \mid \theta, y\right) \\
& =2 \int_{x} k(x, y)^{2} d F(x \mid \theta, y)-2 K(\theta, y)^{2}
\end{aligned}
$$

where $K(\theta, y)=\int_{x} k(x, y) d F(x \mid \theta, y)$. Hence, we can think of the planner as choosing two functions, $k: \mathbb{R}^{2} \rightarrow \mathbb{R}$ and $K: \mathbb{R}^{2} \rightarrow \mathbb{R}$, so as to maximize

$$
\mathbb{E} w=\int_{(\theta, y)}\left\{\int_{x}\left[-(k(x, y)-\theta)^{2}-2 r^{*} k(x, y)^{2}\right] d F(x \mid \theta, y)+2 r^{*} K(\theta, y)^{2}\right\} d G(\theta, y)
$$


subject to the constraint $K(\theta, y)=\int_{x} k(x, y) d F(x \mid \theta, y)$. Setting up the Lagrangian for this problem, taking the first-order conditions for $k(x, y)$ and $K(\theta, y)$, combining the two (so as to get rid of the lagrange multiplier), and using the law of iterated expectations, we get that the following must hold for almost all $(x, y)$ :

$$
\int_{\theta}\left\{-2[k(x, y)-\theta]-4 r^{*} k(x, y)+4 r^{*} K(\theta, y)\right\} d P(\theta \mid x, y)=0
$$

where $P(\theta \mid x, y)$ is the c.d.f. of the conditional distribution of $\theta$ given $x$ and $y$. Using $\alpha^{*}=2 r^{*} /\left(1+2 r^{*}\right)$ and rearranging, the above reduces to (4). Because the maximization problem is concave, this condition is both necessary and sufficient. (For a more detailed derivation, see Angeletos and Pavan, 2006a.)

Proof of Result 3. Using (5), it is easy to see that, for any given strategy $k: \mathbb{R}^{2} \rightarrow \mathbb{R}$, ex-ante welfare is given by

$$
\begin{gathered}
\mathbb{E} w=-\left(1+2 r^{*}\right) \int_{(\theta, y)}\left\{\int_{x}[k(x, y)-K(\theta, y)]^{2} d F(x \mid \theta, y)\right\} d G(\theta, y) \\
-\int_{(\theta, y)}\left\{K(\theta, y)-\theta^{2}\right\} d G(\theta, y) \\
=-\left(1+2 r^{*}\right) \mathbb{E}(k-K)^{2}-\mathbb{E}(K-\theta)^{2},
\end{gathered}
$$

where $\mathbb{E}[\cdot]$ denotes the expectation over $(\theta, y, x)$. Using the fact that, at the equilibrium strategy, $\mathbb{E}[k]=\mathbb{E}[K]=\mathbb{E}[\theta]$, the above reduces to

$$
\mathbb{E} w=-c\left\{\operatorname{Var}(k-K)+\left(1-\alpha^{*}\right) \operatorname{Var}(K-\theta)\right\}
$$

where $c=1+2 r^{*}>0$. Moreover, by (2)-(3), we have

$$
\operatorname{Var}(k-K)=\frac{(1-\alpha)^{2}(1-\delta)}{(1-\alpha+\alpha \delta)^{2}} \sigma^{2} \quad \text { and } \quad \operatorname{Var}(K-\theta)=\frac{\delta}{(1-\alpha+\alpha \delta)^{2}} \sigma^{2}
$$

It follows that

$$
\mathbb{E} w=-c\left\{\frac{\left(1-\alpha^{*}\right) \delta+(1-\alpha)^{2}(1-\delta)}{(1-\alpha+\alpha \delta)^{2}} \sigma^{2}\right\},
$$

from which the result follows. 


\section{References}

[1] Angeletos, George-Marios, and Alessandro Pavan (2004), "Transparency of Information and Coordination in Economies with Investment Complementarities," American Economic Review 94, 91-98.

[2] Angeletos, George-Marios, and Alessandro Pavan (2006a), "Efficient Use of Information, and Social Value of Information," NBER Working Paper ??.

[3] Angeletos, George-Marios, and Alessandro Pavan (2006b), "Optimal Policy for Economies with Dispersed Information," MIT/Northwestern University mimeo.

[4] Angeletos, George-Marios, Guido Lorenzoni, and Alessandro Pavan (2006), "Beauty Contests: Micro-foundations and Policy Implications," MIT/Northwestern University mimeo.

[5] Hellwig, Christian (2005), "Heterogeneous Information and the Benefits of Public Information Disclosures," UCLA mimeo.

[6] Lorenzoni, Guido (2005), "Imperfect Information, Consumers' Expectations and Business Cycles," MIT mimeo.

[7] Mankiw, N.Gregory, and Ricardo Reis (2006), "Sticky Information in General Equilibrium", prepared for the 2006 meeting of the European Economic Association.

[8] Morris, Stephen, and Hyun Song Shin (2002), "The Social Value of Public Information", American Economic Review 92, 1521-1534.

[9] Morris, Stephen, and Hyun Song Shin (2006), "Optimal Communication", prepared for the 2006 meeting of the European Economic Association.

[10] Roca, Mauro (2005), "Transparency and Monetary Policy with Imperfect Common Knowledge," Columbia University mimeo.

[11] Woodford, Michael (2002), "Imperfect Common Knowledge and the Effects of Monetary Policy," in P. Aghion, R. Frydman, J. Stiglitz, and M. Woodford, eds., Knowledge, Information, and Expectations in Modern Macroeconomics, Princeton University Press. 